Federal Reserve Bank of Minneapolis

Research Department Staff Report 255

October 1998

\title{
Complex Eigenvalues and Trend-Reverting Fluctuations
}

\author{
Costas Azariadis*
}

University of California at Los Angeles

\section{James Bullard*}

Federal Reserve Bank of St. Louis

Lee E. Ohanian*

Federal Reserve Bank of Minneapolis

and University of Minnesota

\begin{abstract}
Autoregressions of quarterly or annual aggregate time series provide evidence of trendreverting output growth and of short-term dynamic adjustment that appears to be governed by complex eigenvalues. This finding is at odds with the predictions of reasonably parameterized, convex one-sector growth models, most of which have positive real characteristic roots. We study a class of one-sector economies, overlapping generations with finite life spans of $L \geq 3$, in which aggregate saving depends nontrivially on the distribution of wealth among cohorts. If consumption goods are weak gross substitutes near the steady state price vector, we prove that the unique equilibrium of a life cycle exchange economy converges to the unique steady state via damped oscillations. We also conjecture that this form of trend reversion extends to production economies with a relatively flat factor-price frontier, and we test this conjecture in several plausible parameterizations of 55-period life cycle economies.

* Costas Azariadis thanks the Federal Reserve Bank of St. Louis for hospitality provided during the formative stages of this research. The authors thank Luisa Lambertini, John Laitner, and Harald Uhlig for helpful discussions and Nick Meggos for research assistance. The views expressed in this paper are those of the authors and not necessarily those of the Federal Reserve Bank of St. Louis, the Federal Reserve Bank of Minneapolis, or the Federal Reserve System.
\end{abstract}




\section{Introduction}

Persistence and trend reversion are two widely documented properties of the U.S. output growth rate. Reduced-form representations of time series data suggest that real per capita gross national product (GNP) and related measures of economic activity undergo damped oscillations in response to temporary external shocks, as documented, for example, by Nelson and Plosser (1982), Blanchard and Quah (1989), and Cogley and Nason (1995). This pattern appears in both the autocorrelation and the impulse-response functions of simple GNP autoregressions (ARs), as well as in vector autoregressions (VARs) that include investment, employment, interest rates, and other variables. Temporary responses to total factor productivity, for example, elicit from reduced-form representations of postwar U.S. data the persistent, hump-shaped, self-reversing output reaction displayed in the solid line of Figure 1. This figure, which we reproduce from Cogley and Nason (1995, p. 499), compares the output response of actual data to a transitory shock, represented by the solid line, and of the business cycle model due to Christiano and Eichenbaum (1992), represented by the dotted line. In the data, output accelerates above its trend rate of growth for a few quarters, eventually slowing down to below-average growth and approaching its trend level within five or six years, whereas the model shows monotonic decay. Cogley and Nason (1995) find similar responses for other business cycle models. We conjecture that monotonic decay would also appear in standard versions of the descriptive growth model, the optimum growth model, the overlapping generations (OLG) growth model with two-period life cycles, as well as the infinite-lived extensions of the OLG model due to Blanchard (1985) and Weil (1989). Cogley and Nason (1995, p. 500) comment that "while GNP first rises and then falls in response to a transitory shock, the model generates monotonic decay. Thus the model does not generate 


\section{Figure 1}

Transitory Impulse Response Function

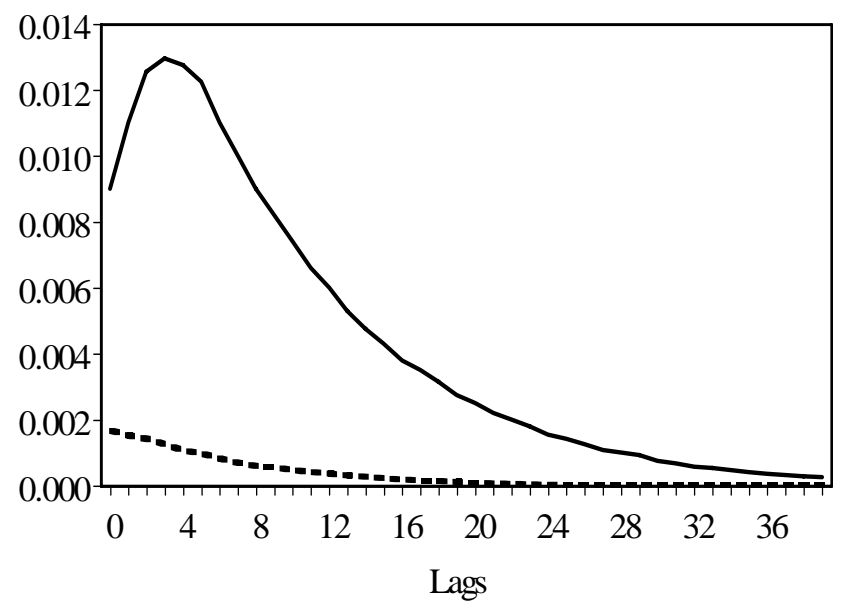

Figure 1: The response of GDP to a transitory shock in the data (solid line) and in a business cycle model (dotted line). In the data, output first grows at a faster-than-trend pace, then at a slower-than-trend pace, following a shock. Eventually output returns to its preshock level. In the model, output only grows at a slower-than-trend pace following the shock. This figure is reproduced from the lower right panel of Figure 3 in Cogley and Nason (1995, pp. 492-511).

an important trend-reverting component in output."

Trend reversion motion poses a serious problem for economic theorists. ${ }^{1}$ It means that complex or negative real eigenvalues appear to govern the short-term dynamics of U.S. output and other aggregates, even though all empirically plausible one-sector models of convex growth that we know of are incapable of reversing their equilibrium motion because all their stable eigenvalues are positive and real. VARs, in short, turn out to imply adjustments to transitory disturbances which differ strikingly from the predictions of all widely used growth models. However, it seems sensible to build models which are consistent with both growth and business cycle facts. ${ }^{2}$

\footnotetext{
${ }^{1}$ We postpone until Section 5 discussing how the temporary impulse reactions of reduced-form VARs differ from those of one-sector growth models in persistence and trend reversion.

${ }^{2}$ This belief is based on the seminal contribution of Kydland and Prescott (1982) and the large literature
} 
One solution to this problem is to complicate or discard some of the basic assumptions underlying convex, neoclassical models of one-sector growth. For example, negative stable eigenvalues occur in one-sector OLG models of endogenous fluctuations if we assume certain types of large income effects as in Grandmont (1985), highly elastic labor supply as in Reichlin (1986), or low elasticities of capital-labor substitution as in Benhabib and Laroque (1988). Boldrin and Montrucchio (1986) demonstrate that a multisector optimum growth model has a negative stable eigenvalue when the rate of time preference is sufficiently high. Complex eigenvalues, conversely, appear in representative agent models with large nonconvexities due to monopolistic competition as in Gali (1996) or technological increasing returns to scale as in Benhabib and Farmer (1994) and in nonclassical growth models with investment, gestation, or production lags as in Samuelson (1939).

None of these extended growth models does a good job of reconciling trend reversion with growth theory, because none seems able to predict complex or negative eigenvalues for empirically plausible choices of taste and technology parameters. OLG models with twoperiod life cycles typically need elasticities of substitution in consumption or production to be far below one in order to generate endogenous fluctuations. Multisector optimum growth economies experience cyclical and chaotic dynamics at rates of time preference corresponding to annual interest rates of $100 \%$ or more, according to Boldrin (1989). And estimates of returns to scale in U.S. production due to Basu and Fernald (1997) raise questions about the scale effects required to extract complex eigenvalues from nonconvex economies with a representative household.

It is hard to resist the conclusion that economic theory has not yet come up with inspired by them. 
a plausible explanation for output trend reversion. What mechanisms convert a temporary external shock to a self-reversing motion? In this paper, we propose an answer to this question by taking a careful look at the dynamics of a relatively unfamiliar class of one-sector growth models - OLG economies with finite life cycles of three or more periods. We focus on this class because, as we will show, economies in this class turn out to have two properties that are unique among growth models: (1) equilibrium dynamics depend nontrivially on the intergenerational distribution of wealth, and (2) our calculations show that most of the stable eigenvalues turn out to be complex. These facts suggest that OLG models with realistic life cycles and standard parameter configurations have, in principle, the ability to duplicate, in a neoclassical context, the short-term business cycle movements of output and other macroeconomic time series.

We proceed as follows. Section 2 sums up the evidence on the eigenvalues of the simplest AR and VAR representations of quarterly aggregate time series. Complex or negative characteristic roots are a fairly robust feature of the data, especially when we include lags of at least two quarters. This finding is in accord with Section 3, which calibrates a variety of OLG economies with and without production for life cycles of 3 and 55 periods. We then turn to providing an explanation for the ubiquity of complex or negative eigenvalues in OLG economies with nontrivial life cycles. Section 4 proves a theorem that rules out monotonic convergence to the unique steady-state for endowment economies and certain classes of production economies whenever consumption goods are weak gross substitutes at price vectors close to the steady-state price vector. Section 5 looks more broadly at the issue of matching the impulse-response functions of VARs with those of theoretical growth models. A summary and a list of extensions make up Section 6 . 


\section{Eigenvalues in AR aggregate time series models}

A large literature analyzes the time series properties of U.S. gross domestic product (GDP) and other aggregate variables, as, for example, in Sims (1980). A widely documented finding is that most variables are not well characterized as first-order linear univariate processes. Instead, the data indicate a richer form of autocorrelation than is consistent with a first-order autoregressive $[\mathrm{AR}(1)]$ model and thus raise the possibility of complex or negative eigenvalues in empirical reduced forms. While the autocorrelation properties of macroeconomic time series have been analyzed in detail in the literature, less is known about the corresponding eigenvalues in these reduced-form models.

In this section, we study the eigenvalues in estimated AR models of GDP and VAR models of GDP and other aggregate time series. We focus on the likelihood that there are complex or negative eigenvalues in the AR and VAR representations of aggregate time series that are routinely used in empirical macroeconomics. We start by estimating simple univariate ARs for real GDP and report the eigenvalues based on the point estimates from the AR. To assess sampling uncertainty in the eigenvalues, we use a nonparametric bootstrap technique to build an empirical distribution. Following the univariate analysis, we consider VARs between real GDP and other variables. We use postwar quarterly data, ranging from 1948:01 to 1997:04. The data include real GDP, consumption, fixed investment, and the interest rate on three-month U.S. Treasury bills. 


\section{A. Eigenvalues in univariate ARs of GDP}

We first consider univariate models of GDP:

$$
A(L) Y_{t}=\varepsilon_{t}
$$

where $A$ is a vector of coefficients in the lag operator $L$ and $Y$ is the natural $\log$ of real GDP. Since there is no consensus on how to decompose economic time series into trend and cyclical components, we use three approaches that have been used in the literature: (1) the linear time trend, (2) linear and quadratic time trends, and (3) the Hodrick-Prescott (HP) filter. For each model of the underlying trend, the cyclical component $\left(y_{t}\right)$ is defined as the difference between the raw data $\left(Y_{t}\right)$ and the estimated trend component $\left(\hat{Y}_{t}\right)$ :

$$
y_{t} \equiv Y_{t}-\hat{Y}_{t}
$$

Once the cyclical component of the data has been extracted, it is necessary to choose the lag order in the ARs. Since several researchers have reported that $\mathrm{AR}(1)$ models are rejected in favor of higher-order models, we begin by estimating an $\operatorname{AR}(2)$ and an $\operatorname{AR}(3)$ model of the cyclical component of real GDP. We estimate the coefficients using OLS. The two eigenvalues $\left(\lambda_{1}\right.$ and $\left.\lambda_{2}\right)$ based on the estimated $\operatorname{AR}(2)$ coefficients are shown in Table 1 , and the three eigenvalues based on the estimated $\operatorname{AR}(3)$ coefficients are shown in Table 2. 


\begin{tabular}{||c|c|c|c||}
\hline \hline \multicolumn{4}{||c||}{ Table 1. Eigenvalues for GDP AR(2) Model. } \\
\hline \hline & Linear & Quadratic & Hodrick-Prescott \\
\hline \hline$\lambda_{1}$ & 0.953 & 0.847 & $0.5823+0.088 i$ \\
\hline$\lambda_{2}$ & 0.367 & 0.444 & $0.5823-0.088 i$ \\
\hline \hline
\end{tabular}

Table 1: Eigenvalues for the AR(2) univariate model for GDP.

\begin{tabular}{||c|c|c|c||}
\hline \hline \multicolumn{4}{||c|}{ Table 2. Eigenvalues for GDP AR(3) Model. } \\
\hline \hline & Linear & Quadratic & Hodrick-Prescott \\
\hline \hline$\lambda_{1}$ & 0.938 & $0.743+0.105 i$ & $0.714+0.348 i$ \\
\hline$\lambda_{2}$ & 0.536 & $0.743-0.105 i$ & $0.714-0.348 i$ \\
\hline$\lambda_{3}$ & -0.186 & -0.246 & -0.350 \\
\hline \hline
\end{tabular}

Table 2: Eigenvalues for the AR(3) univariate model for GDP.

For the $\mathrm{AR}(2)$ process, we find that both eigenvalues are real and positive for the two deterministic trend specifications (specifications 1 and 2), but both are complex for the HP-filtered data. For the AR(3) process, we find that all eigenvalues are either complex or negative for the quadratic and HP-filtered trends, while one eigenvalue is negative for linear detrending. Since the eigenvalues are functions of the estimated AR coefficients, they are subject to sampling uncertainty. To assess this uncertainty, we use a nonparametric bootstrap technique to construct an empirical distribution of the eigenvalues. This involves the following steps. First, we take the residuals from the fitted equations and shuffle their positions using randomly generated numbers from a uniform density. Second, we construct pseudodata, $\left\{\tilde{y}_{t}\right\}_{t=1}^{T}$, using the reordered innovations and the originally estimated AR parameters. We then re-estimate the AR parameters from the pseudodata and use those new parameters to calculate new eigenvalues. By repeating this resampling procedure many times, we can construct a histogram of the empirical eigenvalues.

Based on 500 replications, we found that the eigenvalues were always real and positive 


\begin{tabular}{||c|c|c|c||}
\hline \hline \multicolumn{5}{||c||}{ Table 3. Eigenvalues for Y/C VAR Model. } \\
\hline \hline & Linear & Quadratic & Hodrick-Prescott \\
\hline \hline$\lambda_{1}$ & 1.127 & -0.147 & $0.596+0.263 i$ \\
\hline$\lambda_{2}$ & 1.013 & 0.534 & $0.596-0.263 i$ \\
\hline$\lambda_{3}$ & 0.133 & 0.795 & -0.081 \\
\hline$\lambda_{4}$ & -0.099 & 0.932 & 0.674 \\
\hline \hline
\end{tabular}

Table 3: Eigenvalues for the VAR with GDP and consumption.

\begin{tabular}{||c|c|c|c||}
\hline \hline \multicolumn{5}{||c|}{ Table 4. Eigenvalues for Y/I VAR Model. } \\
\hline \hline & Linear & Quadratic & Hodrick-Prescott \\
\hline \hline$\lambda_{1}$ & 0.977 & 0.451 & $0.713+0.224 i$ \\
\hline$\lambda_{2}$ & 0.497 & 0.892 & $0.713-0.224 i$ \\
\hline$\lambda_{3}$ & 0.687 & -0.033 & 0.329 \\
\hline$\lambda_{4}$ & -0.051 & 0.771 & 0.054 \\
\hline \hline
\end{tabular}

Table 4: Eigenvalues for the VAR with GDP and investment.

for the linear and quadratic trend cases for the $\operatorname{AR}(2)$ specification. For the HP-filtered data, however, eigenvalues were complex in $92.8 \%$ of the trials. For the AR(3) specification, complex or negative eigenvalues were a common feature across all three trend specifications. For the linear trend, $90.6 \%$ of the trials had at least one real negative eigenvalue, and $2.6 \%$ had at least one complex conjugate pair of eigenvalues. For the quadratic trend, $100 \%$ of the trials had complex eigenvalues, and for the HP-filtered trend, $100 \%$ of the trials had at least one real negative eigenvalue and one complex conjugate pair of eigenvalues. To summarize, we found that for univariate representations, complex eigenvalues are a robust feature if HP detrending is used and are robust for the other detrending methods for $\mathrm{AR}(3)$ or higher-order processes. $^{3}$

\footnotetext{
${ }^{3}$ We also analyzed an $\mathrm{AR}(4)$ and an $\mathrm{AR}(5)$ process, and the results were very similar to those of the $\mathrm{AR}(3)$ in terms of the characteristics of the eigenvalues.
} 


\section{B. Eigenvalues in VAR representations of GDP}

We next analyze eigenvalues in VARs using two-variable systems with one lag. We extract the cyclical components of the variables using the same procedure as in the univariate analysis. The variables in the model are GDP with consumption, investment, or the threemonth Treasury bill rate. The eigenvalues based on the VAR coefficients are shown in Tables 3-5. The two-variable systems are denoted $\mathrm{Y} / \mathrm{C}$ for the output-consumption system, Y/I for the output-investment system, and $\mathrm{Y} / \mathrm{R}$ for the output-interest rate system.

As in the univariate analysis, we used the nonparametric bootstrap technique to assess sampling uncertainty of the eigenvalues from the VARs. We found that complex or negative eigenvalues were also a robust characteristic of these low-dimensional VARs. Table 6 summarizes these results by presenting the fraction of draws in which there were either complex or negative eigenvalues in the three systems we studied using the three detrending procedures. We performed 500 draws in the bootstrap analysis.

Table 6 shows that complex or negative roots occurred frequently in the bootstrap analysis. In particular, there were complex or negative roots in the output-interest rate VAR in each of the 500 draws. The only case in which this did not occur virtually every time was the linear-quadratic detrending case in the output-investment VAR, in which 75 percent of the draws had either complex or negative roots. The analysis presented in this section thus suggests that commonly used reduced-form models of major macroeconomic time series, detrended with conventional procedures, produce characteristic roots that are frequently complex or negative. 


\begin{tabular}{||c|c|c|c||}
\hline \hline \multicolumn{4}{|c||}{ Table 5. Eigenvalues for Y/R VAR Model. } \\
\hline \hline & Linear & Quadratic & Hodrick-Prescott \\
\hline \hline$\lambda_{1}$ & 0.349 & 0.422 & $0.746+0.265 i$ \\
\hline$\lambda_{2}$ & $0.936+0.047 i$ & $0.878+0.075 i$ & $0.746-0.265 i$ \\
\hline$\lambda_{3}$ & $0.936-0.047 i$ & $0.878-0.075 i$ & $0.243+0.161 i$ \\
\hline$\lambda_{4}$ & 0.150 & 0.125 & $0.243-0.161 i$ \\
\hline \hline
\end{tabular}

Table 5: Eigenvalues for the VAR with GDP and an interest rate.

\begin{tabular}{||c|c|c|c||}
\hline \hline \multicolumn{4}{||c||}{$\begin{array}{c}\text { Table 6. Frequency of Negative } \\
\text { or Complex Eigenvalues in VARs. }\end{array}$} \\
\hline \hline & Linear & Quadratic & Hodrick-Prescott \\
\hline \hline$Y / C$ & $97.6 \%$ & $98.6 \%$ & $99.8 \%$ \\
\hline$Y / I$ & $92.6 \%$ & $75.2 \%$ & $100.0 \%$ \\
\hline$Y / R$ & $100.0 \%$ & $100.0 \%$ & $100.0 \%$ \\
\hline \hline
\end{tabular}

Table 6: Frequency of complex or negative eigenvalues in VAR estimates, based on bootstrap estimates of sampling uncertainty.

\section{Dynamic adjustment in life cycle economies}

We explore next the characteristic roots of deterministic OLG economies with finite life cycles of $L \geq 3$ periods, identical households within each generation, and time-invariant characteristics (population, preferences, endowment vectors, and technology). If all pairs of dated consumption goods are weak gross substitutes at all price ratios, then, as Kehoe et al. (1991) show, every endowment economy in this class admits no limit cycles; every endowment economy has instead a unique real steady state with unvalued government liabilities and a unique equilibrium sequence converging to that state. ${ }^{4}$ More relevant for business cycle analysis is that local uniqueness holds under very weak assumptions. Kehoe et al. (1991) prove that the equilibrium sequence is unique near the steady state if dated consumption goods are gross substitutes at all price ratios close to the steady state. In either case, uniqueness means that the number of stable eigenvalues exactly equals the number of initial conditions

\footnotetext{
${ }^{4}$ For details, see Section 4.
} 
describing the distribution of wealth among generations at the beginning of time. Another conclusion we can draw from the convergence result is that life cycle economies eventually dissipate external impulses and cannot deviate from their steady state for any prolonged period of time unless they are periodically shocked from outside. Kehoe et al. (1991), however, do not say whether convergence is monotonic or oscillatory. ${ }^{5}$

To sort things out, we compute eigenvalues for a fairly large class of homothetic life cycle economies with and without production for two life cycle lengths: $L=3$ to explore the economic intuition behind the trend-reversal phenomenon and $L=55$ to approximate the year-to-year dynamic adjustment process of a plausibly calibrated economy. In every economy we consider, there is one consumption good, and the trend rate of growth is exactly zero. No technological change or population growth takes place. Each household has utility

$$
U=\sum_{i=0}^{L-1} \beta^{i} \frac{c_{t}(t+i)^{1-\gamma}}{1-\gamma}
$$

where $c_{t}(t+i)$ is the consumption of the agent born in period $t$ in period $t+i$, the discount factor is $\beta>0$, and the curvature parameter $\gamma>0, \gamma \neq 1$, describes the inverse elasticity of intertemporal substitution.

In pure exchange economies, agents receive consumption good endowments $e_{i} \geq 0$ in each period of life $i=1, \ldots, L$ and store value in personal loans. The net supply of these loans is zero. We normalize the community endowment to unity, setting $\sum_{i=1}^{L} e_{i}=1$.

Production economies, on the other hand, use capital and labor to produce a single

\footnotetext{
${ }^{5}$ Auerbach and Kotlikoff (1987) and Huang, İmrohoroğlu, and Sargent (1997) simulate long-term behavior of production economies with a 55-period life cycle and find convergence to be monotonic, possibly due to the capital stock adjustment costs included in their economies. Recursive equilibria, studied by Ríos-Rull (1996), also exhibit monotonic convergence.
} 
consumption good under constant returns to scale. The supply of efficiency labor units is inelastic, denoted by $\omega_{i} \geq 0$ for $i=1, \ldots, L$, and normalized again to sum to one. The stores of value in this economy are loans (in zero net supply) and capital (in positive net supply). Technology is in the CES class; that is,

$$
y(t)=\theta\left[\alpha k(t)^{-\rho}+1-\alpha\right]^{-1 / \rho}
$$

where $y(t)$ is output in period $t, k(t)$ is capital (also the capital-effective labor ratio) in period $t$, the scale parameter $\theta>1$, capital share $\alpha \in(0,1)$, and $\rho>-1$ governs capitallabor substitutability. If $\rho=0$, the technology is Cobb-Douglas. Capital depreciates at the net rate $\delta \in[0,1]$.

If we denote total household assets held in period $t$ by $A(t)$, then the equilibrium condition corresponding to unvalued or nonconstant government liabilities is

$$
A(t)=0
$$

for endowment economies and is

$$
A(t)-k(t+1)=0
$$

for economies with production.

Each economy contains $L$ asset trading generations from the youngest at age $i=0$ to the oldest at age $i=L-1$. Trading plans in period $t$ are conditional on the $2 L-1$ dimensional price vector $p^{t}=\left(p_{t-L+1}, \ldots, p_{t}, \ldots, p_{t+L-1}\right)$. Walras' law and the zero-degree homogeneity of 
excess demands mean that trading plans depend on just $2 L-3$ price ratios. Hence, the equilibrium conditions (5) and (6) are nonlinear difference equations of order $2 L-4$ and $2 L-3$, respectively. These conditions are supplemented by $L-2$ initial conditions in the endowment economy and $L-1$ initial conditions in the production economy, which describe the pre-existing asset and liability positions of households in the initial period $t=0$.

\section{A. Logarithmic endowment economies with three-period lives}

Equilibrium in these economies is any solution to the second-order difference equation

$$
\alpha_{t}(t)+\alpha_{t-1}(t)=0
$$

for $t=1,2, \ldots$, which satisfies the initial condition

$$
\alpha_{0}(0)+\alpha_{-1}(0)=0
$$

In these equations, $\alpha_{t}(t+i)$ denotes claims on other households held by a household of generation $t$ at the end of period $t+i$, for $i=0,1,2, \ldots$ Equation (8) is an initial condition that specifies what the two transitional generations owe each other; it is the equivalent of fixing the interest rate at $t=0$.

As a starting example, we use the logarithmic utility function $u^{t}=\ln c_{t}(t)+\beta \ln c_{t}(t+$ $1)+\beta^{2} \ln c_{t}(t+2)$ for each generation $t=1,2, \ldots$, and the endowment vector $\left(e_{0}, e_{1}, e_{2}\right) \in \mathbb{R}_{+}^{3}$ such that $e_{0}+e_{1}+e_{2}=1$. We start with the asset accumulation identities:

$$
\alpha_{t}(t)=e_{0}-c_{t}(t)
$$




$$
\alpha_{t}(t+1)=R_{t} \alpha_{t}(t)+e_{1}-c_{t}(t+1)
$$

where $R_{t}=p_{t} / p_{t+1}$ is the real interest factor on loans made at $t$ and repaid at $t+1$. The consumer's first-order conditions yield

$$
\begin{aligned}
c_{t}(t+1) & =\beta R_{t} c_{t}(t), \\
c_{t}(t) & =\frac{e_{0}+\frac{e_{1}}{R_{t}}+\frac{e_{2}}{R_{t} R_{t+1}}}{1+\beta+\beta^{2}} .
\end{aligned}
$$

Substitution of (9) and (10) into (7) and (8) yields the second-order equation

$$
\begin{aligned}
x_{t+1} & =R_{t} \\
R_{t+1} & =\frac{\gamma e_{2}}{f\left(x_{t}, R_{t}\right)}
\end{aligned}
$$

where $\gamma=\frac{1}{1+\beta+\beta^{2}}$, and

$$
\begin{aligned}
f(x, R)= & \left\{(1-\gamma) e_{0}+[1-(1+\beta) \gamma] e_{1}+e_{0}[1-(1+\beta) \gamma] x\right\} \\
& \times R-\gamma\left[e_{1}+(1+\beta) e_{2}\right]
\end{aligned}
$$

As we detail in the Appendix, equation (15) turns out to have a unique steady state $\bar{R}$, just as predicted by Kehoe et al. (1991). It also has a characteristic polynomial

$$
\pi(\lambda)=\lambda^{2}-T \lambda+D
$$


with

$$
\begin{aligned}
T & =-\left(\frac{A R^{2}}{\gamma e_{2}}+D\right)<0 \\
D & =\frac{R^{3} e_{0}[1-(1+\beta) \gamma]}{\gamma e_{2}} \\
A & =(1-\gamma) e_{0}+[1-(1+\beta) \gamma] e_{1}
\end{aligned}
$$

and two negative eigenvalues such that

$$
\lambda_{2}<-1<\lambda_{1}<0
$$

Combining the initial condition (8) with the single stable eigenvalue (20), we conclude that real equilibrium is unique in this economy. Whenever the initial distribution of claims $\left\{\alpha_{0}(0), \alpha_{-1}(0)\right\}$ happens to deviate slightly from its steady-state configuration for whatever reason (for example, unexpected policy intervention), the interest rate will also differ from its stationary value $\bar{R}$. Dynamic adjustment to $\bar{R}$ can be locally approximated by the linear equation

$$
R_{t}-\bar{R}=\lambda_{1}\left(R_{t-1}-\bar{R}\right)
$$

in which $-1<\lambda_{1}<0$. The adjustment path shown in Figure 2 shows the oscillatory pattern consistent with reduced-form VARs.

\section{B. More-general endowment economies with $L=3$}

In this section, we explore how robust a negative stable eigenvalue is among exchange economies with a three-period life cycle. To do so, we calculate numerically the eigenvalues 
Figure 2

Qualitative impulse-response.

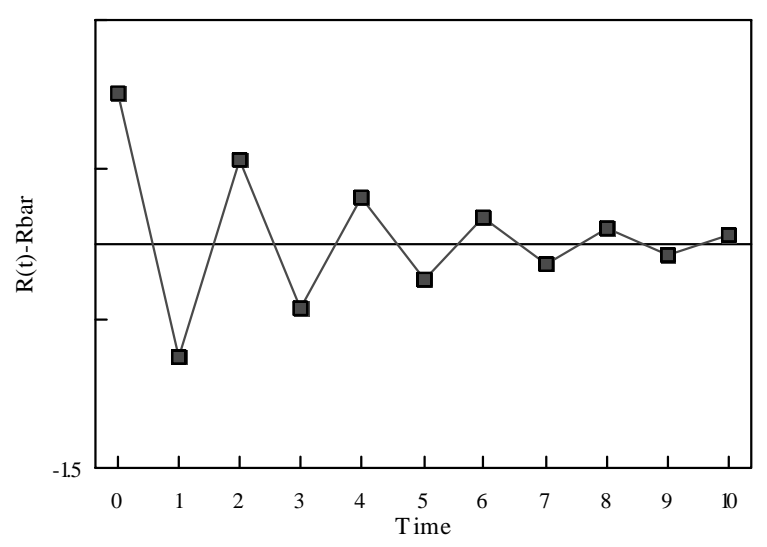

Figure 2: The qualitative impulse-response for a logarithmic preferences life cycle endowment economy with $L=3$.

of large numbers of economies drawn from certain classes and report the results. From this, we gain intuition about the nature of eigenvalue constellations in larger, more interesting models.

Constant Relative Risk Aversion (CRRA) utility with gross substitutes maintained

The logarithmic preferences economy discussed above possesses a single stable eigenvalue which is always real and negative. Our first extension is to study a class of similar economies in which preferences are in the CRRA class, but in which the curvature parameter is restricted to lie between 0 and 1 . By limiting these economies to intertemporal substitution elasticities greater than unity, we preserve the gross substitutes property. We conjecture that the negative stable eigenvalue is always a feature of gross substitutes endowment economies in this class with $L=3$. Preferences are given by (3) with $L=3$. An economy in the set $E_{1}$ is a tuple $\left(\beta, \gamma, e_{1}, e_{2}, e_{3}\right) \in E_{1}$, with $\beta \in(0,1), \gamma \in(0,1), e_{1}+e_{2}+e_{3}=1$, and $e_{i}>0$. An 


\begin{tabular}{||c|c|c|c||}
\hline \hline \multicolumn{3}{||c||}{ Table 7. Eigenvalues of economies in $E_{1}$} \\
\hline \hline & Minimum & Maximum & Median \\
\hline \hline$\lambda_{1}$ & -5353 & -1.918 & -6.57 \\
\hline$\lambda_{2}$ & -0.842 & -0.068 & -0.329 \\
\hline$R$ & 0.303 & 1660 & 2.07 \\
\hline \hline
\end{tabular}

Table 7: Summary statistics for eigenvalues and the interest rate of 1,000 randomly selected economies in $E_{1}$. This class of economies is large in the sense that discount factors near zero and endowments near zero and in arbitrary sequence are allowed.

economy is randomly selected from $E_{1}$ as follows. The preference parameters $\beta$ and $\gamma$ are randomly selected from uniform distributions on the unit interval. For the endowments, we select three random numbers from uniform distributions on the unit interval, say, $r_{1}, r_{2}, r_{3}$. We then create an endowment pattern by setting $e_{i}=\frac{r_{i}}{r_{1}+r_{2}+r_{3}}$. This procedure encompasses a wide class of possible endowment patterns. With an economy in hand, we calculate the steady state and then find the associated eigenvalues. We repeat this process to create a sample of 1,000 economies in $E_{1}$.

The results shown in Table 7 strengthen our conjecture about the relationship between the negative stable eigenvalue and the gross substitutes property. In particular, the eigenvalues for the 1,000 economies in $E_{1}$ are always real and negative. One of these eigenvalues is always outside the unit circle, while the other is inside. Thus, adjustment to the steady state following a temporary disturbance is always damped and oscillatory for these economies. We also report the steady-state interest rate for these economies, which can be either less than or greater than unity, indicating for the former cases that these economies can sometimes be inefficient. However, this fact does not affect the qualitative local dynamics around the steady state. 


\section{Additional curvature}

If we allow significant curvature in preferences, the gross substitutes property does not necessarily hold and the uniqueness of steady state equilibrium can be lost. However, we want to explore how our results from the preceding subsection change if $\gamma>1$. To reduce clutter and report the most interesting features, we also restrict the remaining parameters. Accordingly, an economy in $E_{2}$ is a tuple $\left(\beta, \gamma, e_{1}, e_{2}, e_{3}\right) \in E_{2}$ in which the following restrictions apply. We choose from a uniform distribution $\gamma \in(0,3)$, which implies intertemporal elasticities of substitution as small as $\frac{1}{3}$. The discount factor is chosen from a uniform distribution on $(0.5,2)$, corresponding to rates of time preference that might be viewed as realistic given the length of the time period. The endowment sequence is restricted to be hump-shaped with $e_{1}=$ $e_{3}$ and the middle endowment chosen from a uniform distribution with $e_{2} \in\left(e_{1}, 3 e_{1}\right)$. This pattern corresponds roughly to data sometimes used to calibrate larger models, in which the peak endowment is about 1.7 times the first endowment and the final endowments are of about magnitude equal to the initial endowments. We maintain the restriction that $e_{1}+e_{2}+e_{3}=1$. We randomly select 1,000 economies from $E_{2}$ and calculate the steady state as well as the associated eigenvalues.

The results are shown in Figure 3. These economies turn out to always have a unique steady state, and they continue to possess a single stable eigenvalue. This stable eigenvalue, however, can now sometimes be positive. In Figure 3, we plot the value of the stable eigenvalue for each of our 1,000 sample economies against four characteristics of the economy. In Panel $\mathrm{A}$, the value of the stable eigenvalue is plotted against $e_{2}$, which can be interpreted as the peakedness of the endowment pattern. It is clear that flatter endowment sequences tend to preserve the negative sign on the stable eigenvalue. Similarly, Panels B and D show 
Figure 3. How the negative stable eigenvalue relates to properties of endowment economies.
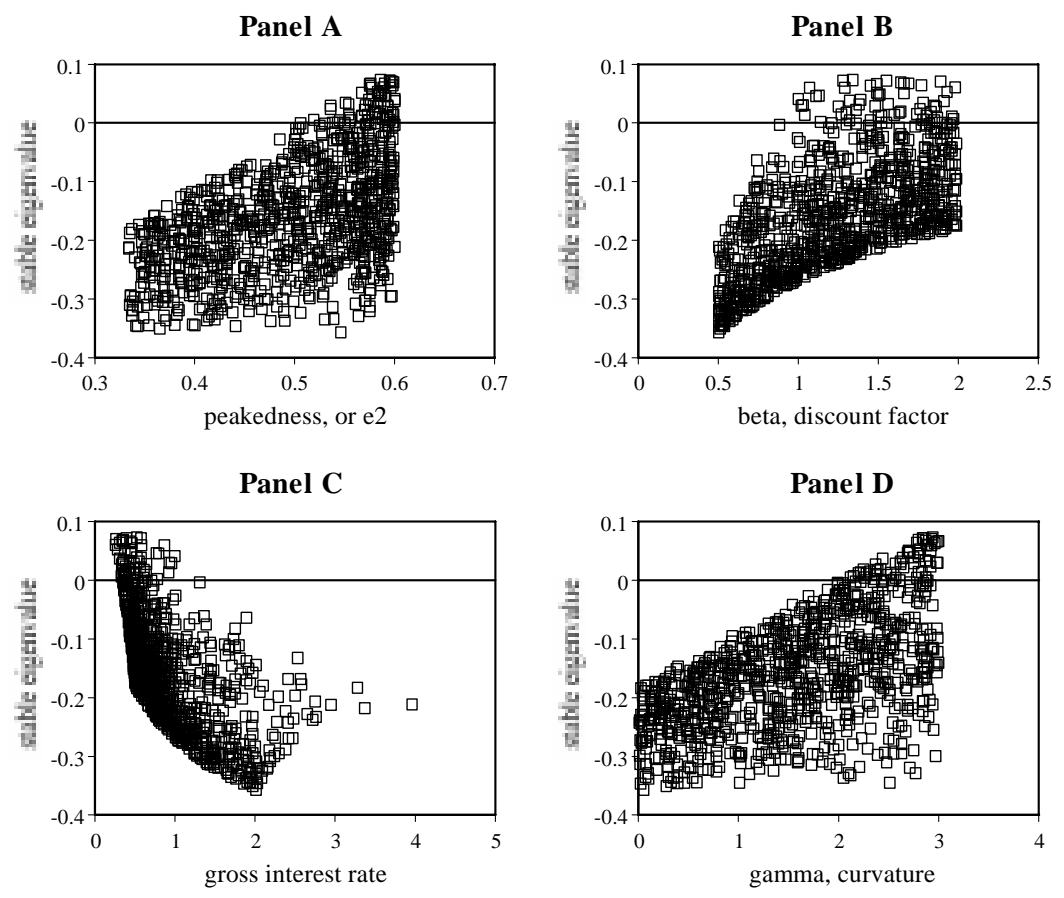

Figure 3: The stable eigenvalue for the 1,000 economies in $E_{2}$ is typically negative. The four panels relate the stable eigenvalue to characteristics of these economies.

that discount factors less than unity and curvature parameters less than two tend to preserve the negative sign on the stable eigenvalue, regardless of other parameters. Panel $\mathrm{C}$ relates the value of the stable eigenvalue to the steady-state gross interest rate. Here, we see that the inefficient economies, those with gross interest rates less than unity, can be characterized by a positive stable eigenvalue. These results suggest that oscillatory adjustment to transitory shocks can characterize efficient economies with empirically plausible features: positive rates of time preference, relatively flat endowment profiles, and relatively high elasticities of intertemporal substitution (above $\frac{1}{2}$ ). 


\section{Production economies with three-period life cycles}

In economies with three-period life cycles and production, the dimension of the associated dynamic system increases by one. Initial conditions are now the holdings of capital owned by the agents who have been alive for one and two periods. For determinacy to hold, we expect two stable eigenvalues in these systems. We use the same CRRA utility function and the CES production function given by equation (4). Labor supply is inelastic and normalized to unity. An economy in the set $E_{3}$ is a tuple $\left(\beta, \gamma, e_{1}, e_{2}, e_{3}, \alpha, \rho, \delta\right)$, where $\alpha$ is capital share, $\rho$ is the inverse of the elasticity of substitution between capital and labor, and $\delta$ is the net rate of depreciation for physical capital. For the preference parameters, we choose from uniform distributions $\beta \in(0.5,2)$ and $\gamma \in(0.5,3)$. We use the hump-shaped endowment pattern described in the preceding section. For the production parameters, we choose, again, from uniform distributions, $\alpha \in(0.25,0.4), \rho \in(-0.5,0.5)$, and $\delta \in(0.8,1)$. We set the scale parameter $\theta$ to 10 , which is sufficient to guarantee existence. We calculate the steady state and the associated eigenvalues.

The 1,000 economies in $E_{3}$ always possess a unique, determinate steady state. In each case, we find that one stable eigenvalue is real and positive and the other is real and negative. The modulus of the two eigenvalues is of similar magnitude in most cases, but the positive eigenvalue is larger in absolute value in about $85 \%$ of the economies. Figure 4 shows our findings and relates the characteristics of the economies to the magnitude of the two stable eigenvalues. The results here are much less ambiguous than those for the economies in $E_{2}$. Here, the negative eigenvalue is always present and can be dominant under many parameter configurations. 
Figure 4. How the stable eigenvalues of 3-period production economies relate to economic characateristics
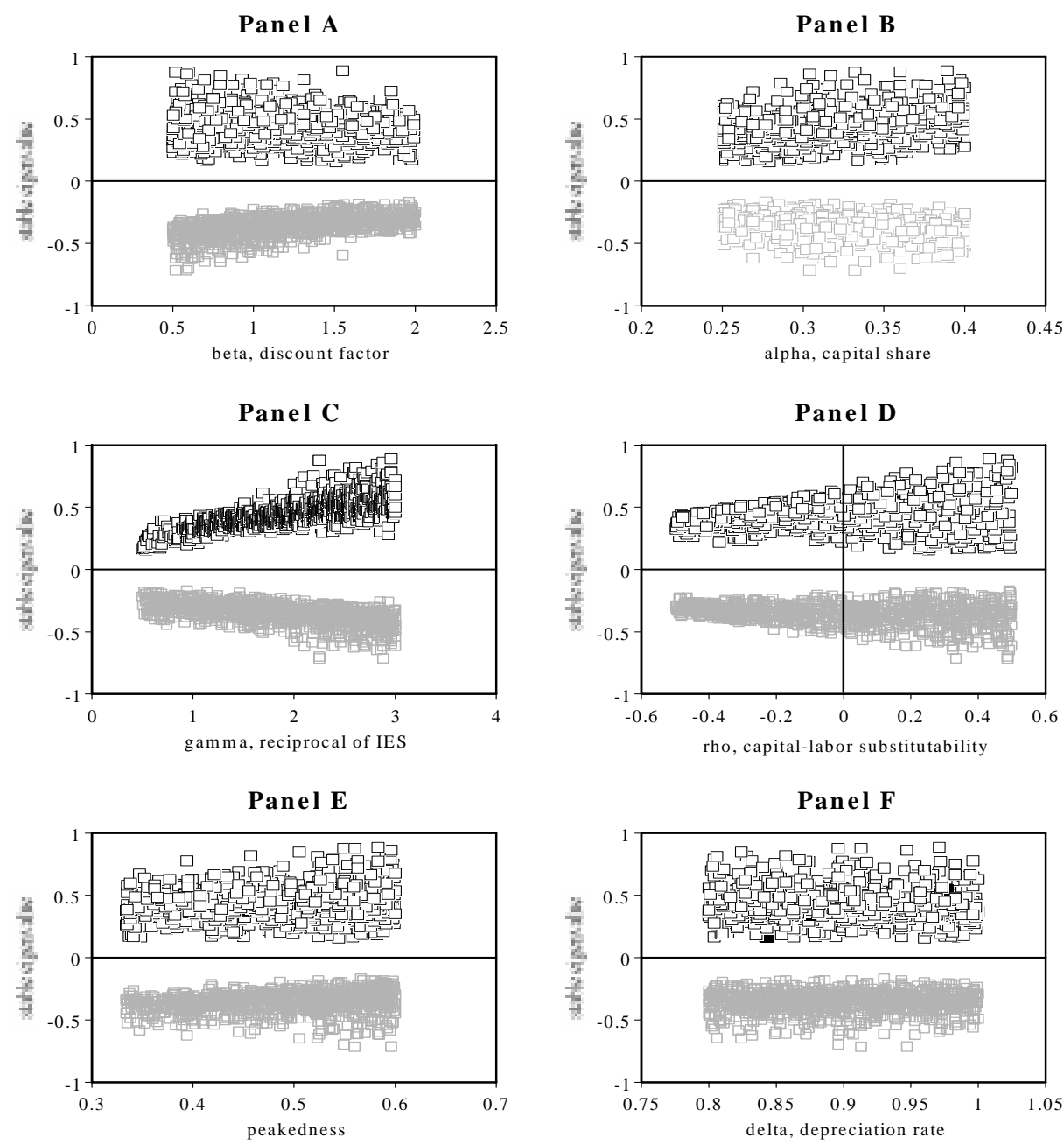

Figure 4: The two stable eigenvalues of three-period production economies, related to characteristics of these economies. 


\section{Production economies with 55-period life cycles}

We ultimately want to understand the local dynamics of life cycle economies with many periods, in which agents are allowed to make decisions and thus react to shocks at many points in their lives. Accordingly, we study economies with $L=55$, annual models. Here we calibrate the economies much more sharply in order to reduce the number of cases we need to calculate. Our calibration proceeds as follows. We use a productivity profile based on Hansen (1993). ${ }^{6}$ We set the following parameters at annualized values: $\beta=0.98$, $\delta=0.065$. We set capital share $\alpha=0.33$. That leaves two parameters, curvature in preferences and capital-labor substitutability, which can be viewed as the most interesting parameters for dynamic adjustment. We explore nine cases based on the following $(\rho, \gamma)$ pairs: $A=$ $(-0.5,1.1), B=(-0.5,2), C=(-0.5,5), D=(0,1.1), E=(0,2), F=(0,5), G=(0.5,1.1)$, $H=(0.5,2)$, and $I=(0.5,5)$. Accordingly, case $D$ is close to a $\log -\log$ specification, in which preferences are logarithmic and production is Cobb-Douglas. ${ }^{7}$ For each case $A, \ldots, I$, we calculate the steady state of the system. In principle, uniqueness of the steady state is not guaranteed, but multiple steady states do not occur for these parameter configurations. There is also the question of whether the calculated steady states are efficient or inefficient. The gross growth rate in these economies is one, so any gross interest rate greater than one indicates that the equilibrium is efficient. Steady-state interest rates are always greater than

\footnotetext{
${ }^{6}$ The Hansen data are collected from samples taken in 1979 and 1987. The data separate males from females. We average the data from the two years, and we also average the data across males and females using weights of 0.6 and 0.4. The resulting profile is a step function, because the data are collected for age groupings. We fit a fifth-order polynomial to this step function. This yields the smooth profile $e_{i-20}$ $=m_{0}+m_{1} i+m_{2} i^{2}+m_{3} i^{3}+m_{4} i^{4}+m_{5} i^{5}$ for $i=21, \ldots, 76$, with the vector of coefficients $m=[-4.34$, $\left.0.613,-0.0274,0.0063,-0.717 \times 10^{-5}, 0.314 \times 10^{-7}\right]$. This profile peaks at agent age 28 (figurative age 48 ), when productivity is about 1.6 times its level at agent age 1 (figurative age 21). Productivity in the final year of life is virtually the same as in the first year of life.

${ }^{7}$ To avoid special programming code, we did not allow the case in which $\gamma$ is exactly equal to unity. This makes little difference for the results.
} 
Figure 5

Eigenvalues, 55-period model

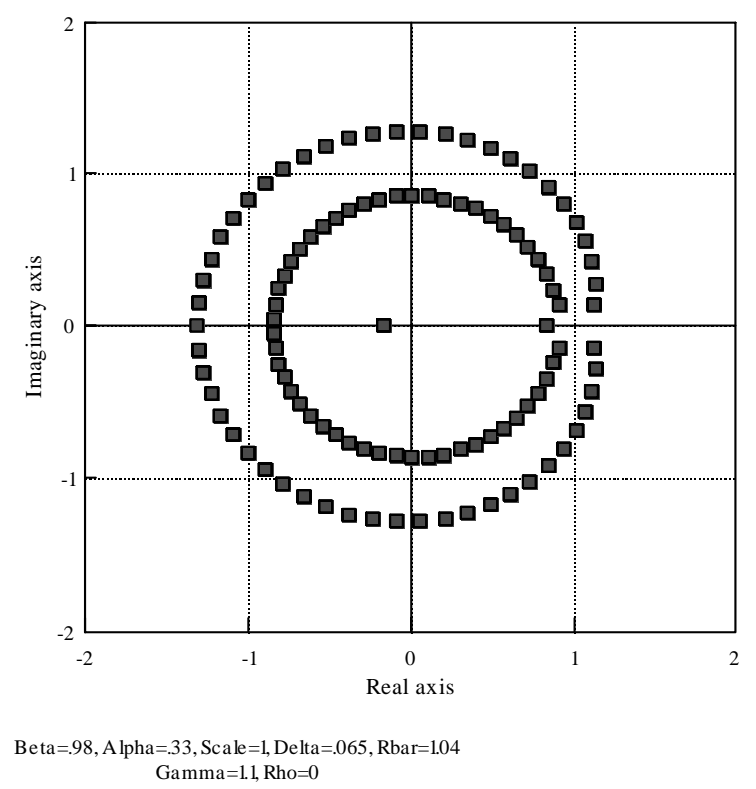

Figure 5: The eigenvalues for Case $D$. The eigenvalues in the inner loop are all inside the unit circle. Since there are 54 of them, the economy is determinate. The cases $A, \ldots, I$ all produced qualitatively similar diagrams.

one for each of the nine cases $A, \ldots, I$, so we are looking at only efficient economies.

We study the local dynamics of these economies. In Figure 5, we plot the associated eigenvalues in the complex plane for case $D$. Each square plotted represents an eigenvalue associated with the unique steady state. The eigenvalues on the inner loop are all less than unity in modulus, and since there are exactly 54 of them, the equilibrium is determinate. Perhaps more importantly for our purposes, nearly all the roots are complex and of about equal modulus. ${ }^{8}$ Thus, we expect the local dynamics of these systems to be characterized by fluctuating motion. The qualitative features of this figure are the same for the other nine

\footnotetext{
${ }^{8}$ Laitner (1990) calculates eigenvalues for a small sample of alternatively parameterized large life cycle economies and finds complex eigenvalues. In those economies, taxes play an important role.
} 


\begin{tabular}{||c|c|c|c||}
\hline \hline \multicolumn{4}{|c|}{ Table 8. Comparison of cases $A, \ldots, I}$. \\
\hline \hline Economy & Minimum & $2^{\text {nd }}$ Smallest & Largest Stable \\
\hline$(\rho, \gamma)$ & Modulus & Modulus & Modulus \\
\hline \hline$A=(-.5,1.1)$ & 0.153 & 0.856 & 0.944 \\
\hline$B=(-.5,2)$ & 0.049 & 0.881 & 0.956 \\
\hline$C=(-.5,5)$ & 0.003 & 0.833 & 0.993 \\
\hline$D=(0,1.1)$ & 0.163 & 0.837 & 0.930 \\
\hline$E=(0,2)$ & 0.104 & 0.855 & 0.939 \\
\hline$F=(0,5)$ & 0.017 & 0.777 & 0.981 \\
\hline$G=(.5,1.1)$ & 0.164 & 0.759 & 0.932 \\
\hline$H=(.5,2)$ & 0.122 & 0.797 & 0.937 \\
\hline$I=(.5,5)$ & 0.033 & 0.744 & 0.973 \\
\hline \hline
\end{tabular}

Table 8: Summary of eigenvalues for cases $A, \ldots, I$.

cases - we always find two groups of eigenvalues, one group lying roughly evenly spaced on an ellipse outside the unit circle and the other lying roughly evenly spaced on an ellipse inside the unit circle. The idea that the eigenvalues are roughly evenly spaced can be documented if we translate to polar coordinates and measure the distance between eigenvalues in degrees. In this metric, the stable eigenvalues are all about 360/54 degrees apart, the only substantial exceptions occurring near the point $(1,0)$ in the diagram, where the roots are slightly farther apart. Of the two stable real roots one has modulus comparable to the complex roots and the other is relatively small and negative. We summarize the results for the nine cases in Table 8 .

\section{Trend reversion and gross substitutes}

Simulations from Section 3 suggest that gross substitutes tend to rule out monotonic convergence - specifically, if all pairs of dated consumption goods are weak gross substitutes in the neighborhood of the steady-state price vector, then equilibria near the steady-state exhibit damped oscillations for all exchange economies with a life cycle $L \geq 3$ and for some 
production economies as well.

Under gross substitutes, aggregate asset demand - which must vanish in an exchange equilibrium - is an increasing function of all relevant interest rates. This technical result means roughly that all $L-1$ nontransitional households will keep building their assets as long as interest rates remain above the steady state, and the resulting asset gain must be exactly offset by the reduction in asset holdings of the lone transitional generation - the oldest one alive. For a large value of $L$ - that is, a short trading period - monotonic decay toward the steady state would seem to require an enormous stock of assets in the hands of the oldest living cohort.

Intuition supporting the conjecture of damped oscillations is easier to conjure up in an exchange economy with the typical peaked endowment pattern. Consumption smoothing in this environment produces a steady state in which the young borrow from the middle aged, repay loans and build up assets in middle age, and draw down these assets in old age. If the interest rate at $t$ is above its steady state value $\bar{R}$, then young agents postpone consumption and reduce their liabilities. Middle-aged agents, according to equation (7), must correspondingly reduce their asset holdings even as they are shifting consumption away from the current period $t$. This is a consistent course of action for them only if the middle aged reduce current consumption to repay unusually high debts carried from the previous period $t-1$. Unusually high consumption in youth is rational only if the interest rate was unusually low at $t-1$. Hence, $R_{t}>\bar{R}$ implies that $R_{t-1}<\bar{R}$. 


\section{A. Convergence in exchange economies}

Formal results come from studying a double-ended deterministic exchange economy with finite life cycles of length $L=T+1 \geq 3$, constant population, and one agent in each cohort. Our economy is very similar to the one in Kehoe et al. (1991). We denote time periods by $t=0, \pm 1, \pm 2, \ldots$, and cohorts by $\nu$. Each cohort has a stationary, nonnegative endowment vector $e^{\nu}=\left\{e_{\nu+i}^{\nu}\right\}_{i=0}^{T}=\left\{e_{i}\right\}_{i=0}^{T}$, a consumption vector $c^{\nu}=\left\{c_{\nu}(\nu+i)\right\}_{i=0}^{T}$, and an additive utility function $U^{\nu}=\sum_{i=0}^{L-1} \beta^{i} u\left[c_{\nu}(\nu+i)\right], \beta>0$, satisfying standard smoothness, monotonicity, and convexity properties.

The price vector relevant to cohort $\nu$ is $p^{\nu}=\left\{p_{t}\right\}_{t=\nu}^{\nu+T}$, which implies price ratios or interest rates $\left\{R_{t}\right\}_{t=\nu}^{\nu+T}$ with $R_{t}=p_{t} / p_{t+1}$. Maximizing utility subject to the budget constraint $p^{\nu}\left(c^{\nu}-e^{\nu}\right) \leq 0$ leads to asset demand schedules of the form

$$
\begin{aligned}
\alpha_{\nu}(t) & =\sum_{i=\nu}^{t}\left(\frac{p_{i}}{p_{t}}\right)\left[e_{i-\nu}-c_{\nu}(i)\right] \\
& \equiv z\left(p^{\nu} / p_{t} ; t-\nu\right) .
\end{aligned}
$$

These schedules satisfy standard accounting and budget constraints; that is,

$$
\alpha_{\nu}(t)=R_{t-1} \alpha_{\nu}(t-1)+e_{t-\nu}-c_{\nu}(t)
$$

where $t=\nu+1, \ldots, \nu+T-1$ and

$$
\alpha_{\nu}(\nu-1)=0=\alpha_{\nu}(\nu+T)
$$


For each stage of the life cycle $t-\nu=0, \ldots, T$, the asset demands are homogeneous of degree zero in the vector $p^{\nu}$, depending only on the vector $p^{\nu} / p_{t}=\left(p_{\nu} / p_{t}, \ldots, p_{\nu+T} / p_{t}\right)$ for any cohort $\nu$ and any time period $t=\nu, \ldots, \nu+T$. Since price ratios are products of interest factors, we can rewrite real asset demands as functions of these interest factors; that is,

$$
\alpha_{\nu}(t)=z^{\star}\left(R_{\nu}, \ldots, R_{\nu+T-1} ; t-\nu\right)
$$

Gross substitutability implies that each asset demand schedule is monotonic in the vector of interest factors. We prove in the Appendix the following result:

LEMMA 1. (Monotonicity.) If all pairs of dated consumption goods are weak gross substitutes for cohort $\nu$, then $\alpha_{\nu}(t)$ is an increasing function of the vector $\left(R_{\nu}, \ldots, R_{\nu+T-1}\right)$ for each $t=\nu, \ldots, \nu+T-1$

Monotonicity easily extends to the aggregate real asset demand schedule at time $t$ :

$$
A(t)=\sum_{\nu=t-T+1}^{t} \alpha_{\nu}(t) \equiv z^{\star}\left(R_{t-T+1}, \ldots, R_{t+T-1}\right)
$$

which depends positively on a $2 T-2$ dimensional vector of interest factors.

This economy admits a unique stationary real equilibrium but no periodic real equilibria. Stationary equilibria are constant interest rate sequences $R_{t}=R^{\star}>0$ or geometric price sequences $p_{t}=p_{0}\left(R^{\star}\right)^{-t}$, satisfying $A(t)=0$ for $t=0, \pm 1, \pm 2, \ldots$. The increasingness of the schedule $z^{\star}$ rules out multiple steady states, and Kehoe et al. (1991, pp. 13-15) demonstrate that one such state exists by bounding $z^{\star}$ from above and below. We sum up in the following: 
LEMMA 2. (Uniqueness of the steady state.) Weak gross substitutes imply that a unique nonmonetary steady state exists.

Of immediate concern to us are high-frequency adjustments to temporary external shocks at, say, $t=0$. To study the adjustment process, we look at how the economy evolves from $t=1$ onward either after an unexpected shock that disturbs a stationary equilibrium at $t=0$ or after an anticipated policy intervention that fixes wealth for all generations at the end of period $t=0$. In either case, a nonstationary equilibrium sequence $\left\{R_{t}\right\}_{t=1}^{\infty}$ satisfies $A(t)=0$, $t=1,2, \ldots$, plus $T-1$ independent initial conditions which fix the wealth of all pre-existing generations at the end of period $t=0$. These initial conditions fix $\left\{\alpha_{1-T}(0), \ldots, \alpha_{0}(0)\right\}$ and, in addition, constrain $\sum_{\nu=1-T}^{0} \alpha_{\nu}(0)=0$. The last equation means that transitory generations $\nu=1-T, \ldots, 0$ hold claims against each other only, not against cohorts born at $t=1$ or later. Nonstationary equilibria are solutions to the difference equation $A(t)=0$, which has order $2 T-2$ or $2 L-4$ in the price vector $\left\{R_{t-T+1}, \ldots, R_{t+T-1}\right\}$, subject to the $T-1$ initial conditions. Kehoe et al. (1991, pp. 6-7, 18) prove that there can be no more than one such solution for each economy.

Lemma 3. (Uniqueness of equilibrium.) Under weak gross substitutability, there is at most one equilibrium price sequence which, if it exists, converges to the steady state.

Two corollaries of this result are that limit cycles cannot exist when consumption goods are gross substitutes at all price ratios and that limit cycles cannot exist near the steady state if gross substitutability obtains at prices near the steady state-price vector. More relevant for our purposes is the added implication that the steady-state $R^{\star}$ has $T-1$ unstable eigenvalues with modulus larger than one and $T-1$ stable eigenvalues with modulus smaller than one. 
Convergence to $R^{\star}$ will take place on the stable manifold of this economy, a $T-1$ dimensional subspace defined near $R^{\star}$ by the eigenvectors which correspond to the stable roots.

Trend reversion requires that deviations from the steady state die out as damped oscillations, not as monotonically decaying motions. None of the preceding results say whether the $T-1$ stable eigenvalues are complex or negative, and it would seem very hard to extract such information about the nature of these roots from first principles. The main theoretical result of this section is that convergence to $R^{\star}$ involves some damped oscillatory motion. Formally, we have the following:

Theorem 1. If dated consumption goods are weak gross substitutes at price ratios near $R^{\star}$, then the unique equilibrium price sequence $\left\{R_{t}^{\star}\right\}_{t=1}^{\infty}$, if it exists, cannot be monotonic.

Proof. Monotonic convergence implies that for all $t \geq \tau$, either $R_{t}^{\star}>R^{\star}$ or $R_{t}^{\star}<R^{\star}$. In view of equation (27), the first alternative means that $A(s)>0$ for all $s \geq \tau+T-1$. The second alternative means that $A(s)<0$ for all $s \geq \tau+T-1$. Both implications violate the equilibrium condition $A(t)=0$.

We conclude that some of the $T-1$ stable roots associated with the steady state $R^{\star}$ must be complex or negative and that adjustment in the neighborhood of $R^{\star}$ is dominated by these eigenvalues. 


\section{B. Convergence in production economies}

The equilibrium of a production economy with a life cycle of $L \geq 2$ periods satisfies a difference equation of order $2 L-3$, which represents zero aggregate excess demand for assets,

$$
A(t)-k(t+1)=0,
$$

plus $L-1$ independent initial conditions fixing the wealth of transitional generations, that is, $\left\{\alpha_{1-T}(0), \ldots, \alpha_{0}(0)\right\}$ given $\sum_{\nu=1-T}^{0} \alpha_{\nu}(0) \equiv k_{1}>0$. Despite the similarities between this economy and the preceding one, Lemma 3 and Theorem 1 do not extend directly to economies with production. Calvo (1978) and Kehoe (1985), in particular, provide examples of nonunique equilibria in economies with gross substitutability in consumption and a high degree of complementarity in production. We recall also from Section 3 the simulations of the OLG growth model with a three-period life cycle summarized in Figure 4. In that figure, the stable eigenvalue with the largest modulus can be either positive or negative, even for utility functions satisfying $\gamma<1$, that is, functions for which consumption goods are gross substitutes at all price ratios. Hence, gross substitutability is not sufficient to rule out monotonic convergence in OLG growth models with nontrivial life cycles.

The logic of these counterexamples to uniqueness and trend reversion is not entirely clear, but seems to rest on the correlation of prices with incomes as an economy adjusts toward its steady state. In endowment economies, incomes are fixed and uncorrelated with prices or interest rates. In production economies, however, interest rates and wage incomes are negatively correlated by the factor-price frontier.

Deviations from the steady state affect interest rates and wages in opposite directions 
and exert two conflicting forces on savings plans: a higher-than-normal interest rate tends to postpone current consumption and raise asset holdings, while a lower-than-normal wage rate (and wage income) will lower assets as the household borrows against future earnings to smooth out its consumption path. Dynamic adjustment in production economies seems to depend on the balance of these two conflicting forces, that is, on the steepness of the factor-price frontier near the steady state. We conjecture that production economies with relatively flat factor-price frontiers will behave like endowment economies.

\section{Matching models with data}

Comparing the tables of Section 2 with the simulations of Section 3 reveals remarkable qualitative similarities in the dynamic adjustment pattern of aggregate time series autoregressions with that of life cycle economies. VARs and life cycle models display a persistent, self-reversing response to temporary output and interest-rate shocks. The dominant eigenvalues of HP-filtered and quadratic filtered quarterly VARs in Tables 2, 4, and 5 are complex with modulus in the intervals $[0.75,0.80]$ and $[0.75,0.90]$, respectively. The characteristic roots of empirically plausible simulations of 55-period OLG economies are also complex with modulus between 0.80 and 0.98 . As a benchmark, we note that the unique stable eigenvalue of the most basic optimal growth model is 0.975 for quarterly time periods. ${ }^{9}$

When adjustment to external stocks is dominated by a pair of complex eigenvalues $\gamma \pm j \mu$, as it is in both OLG models and autoregressions of postwar U.S. aggregate time

\footnotetext{
${ }^{9}$ This represents the stable eigenvalue of a model with a fixed labor supply, no adjustment costs of any type, homothetic instantaneous utility $u(c)=(1-\gamma)^{-1} c^{1-\gamma}$, Cobb-Douglas technology $f(k)=k^{\alpha}$, and constant capital depreciation and utility discount rates $(\delta, \beta)$, respectively. We close the parameterization $(\alpha, \rho, \gamma, \delta)=$ $(0.33,0.01,2,0.02)$ and the time unit to be a quarter. The stable eigenvalue is the only root of the polynominal $\pi(\lambda)=\lambda^{2}-T \lambda+D$ in the interval $(0,1)$ for $1 / \beta \equiv 1+\rho, D \equiv 1+\rho, T=2+\rho+\theta, \theta \equiv \frac{(1-\alpha)(\rho+\delta)}{\gamma(1+\rho)}\left(\frac{\rho+\delta}{\alpha}-\delta\right)$. When $\rho$ and $\delta$ are smaller than 0.05 , the stable eigenvalue is well approximated by $1-\sqrt{\theta}$.
} 
series, the impulse response of a deviation from a stationary trend subject to initial conditions $\left(x_{0}, x_{1}\right)=(0,1)$ takes the form

$$
x_{t}=r^{t-1} \frac{\sin \theta t}{\sin \theta}
$$

where $r$ is the modulus of each root; that is, $r \equiv\left(\gamma^{2}+\mu^{2}\right)^{1 / 2}$ and $\cos \theta \equiv \gamma / r$.

Any sequence $\left\{x_{t}\right\}_{t=0}^{\infty}$ conforming to equation (5.1) displays positive autocorrelation at high frequencies (that is, $x_{t} x_{t+1}>0$ ) and trend reversion twice in each cycle (that is, $x_{t} x_{t+1}<$ 0). The natural question to ask next is, Can we develop qualitative similarities between life cycle economies and AR representations of aggregate time series into a quantitative match? Is there, in particular, a realistic parameterization of an OLG economy that will duplicate the autocorrelation, spectral density, and impulse-response functions of reduced-form VARs?

Duplicating the evolution of U.S. output at cyclical frequencies has proved to be a difficult job for any growth model, as documented, for instance, by Burnside, Eichenbaum, and Rebelo (1993), Cogley and Nason (1995), and Rotemberg and Woodford (1996). Models based on one-sector optimum growth and one-sector infinite life cycle overlapping generations tend to display persistence and no trend reversion because their dominant eigenvalues are positive. Temporary output shocks decay monotonically and have an implied half-life that varies from 2 to 20 quarters. Persistence can be changed by tweaking some parameters, for example, by adding adjustment costs to capital or labor, lowering the depreciation rate, or raising the elasticity of intertemporal labor substitution. ${ }^{10}$ As we explained in Section 1 , trend reversion cannot occur without major departures from normal parameter values.

\footnotetext{
${ }^{10}$ All these added features slow during the adjustment of the capital-labor ratio to its steady state, either by reducing the elasticity of saving to deviations of the capital-labor ratio from the steady state or by generating labor supply movements in the same direction.
} 
Both persistence and trend reversion are built into life cycle economies when consumption goods are weak gross substitutes and productive factors have a relatively flat factor-price frontier. These qualitative features stem from the interaction between aggregate saving behavior and the distribution of wealth over consumer cohorts. At any point in time, aggregate consumption in a life cycle economy reflects the distribution of permanent income among active households, not just aggregate permanent income. When that distribution fluctuates, so will economic aggregates.

Matching life cycle models with data seems to us quite a challenge. For example, the simulations of 55-period models in Section 3 lead us to believe that OLG models have too much persistence, even with no intertemporal substitutability in the labor supply. Their dominant eigenvalues imply that temporary shocks have exceptionally large half-lives ranging from 12 to 32 quarters. Another problem is the computational complexity of a quarterly life cycle model with positive intertemporal elasticity of substitution and a list of state variables running in the hundreds. Finally, explaining the hump-shaped nature of impulse responses may prove as much of a problem for life cycle models as it has been for representative agent models. The hump shape signals an amplified impulse, possibly due to a nonconvexity or indivisibility in factor markets. ${ }^{11}$

One useful step would be to compare in detail how the autocorrelation and impulseresponse functions implied by U.S. output ARs match those of a calibrated 55-period OLG economy.

\footnotetext{
${ }^{11}$ Bernanke and Gertler (1989) and Ramey and Watson (1997) study how the indivisibility of investment projects and jobs can amplify external shocks in economies with private information. Azariadis and Chakraborty (1998) explore amplification in economies with increasing returns to scale in financial intermediation.
} 


\section{Conclusions}

Convex life cycle economies with pure exchange or with production under constant returns to scale are the simplest class of models consistent with the trend-reverting behavior of U.S. output. This behavior is documented by reduced-form VARs in their response to temporary impulses and in their characteristic roots, which tend to be overwhelmingly complex or negative. Complex eigenvalues also occur in OLG economies with pure exchange when dated consumption goods are gross substitutes near the steady state and aggregate saving depends nontrivially on the distribution of household wealth among successive cohorts of individuals. We conjecture that an array of complex eigenvalues is a likely feature of all life cycle economies with a reasonably large number of decision points in the life cycle. These complex eigenvalues are of comparable modulus to the largest real root.

The qualitative similarity between life cycle economies and VARs in their dynamic adjustment paths naturally brings to the fore the question of quantitative fit. Are there empirically plausible parameterizations of OLG economies in which eigenvalues, autocorrelation functions, and responses to temporary productivity or liquidity shocks match quantitatively those of fitted VARs? Section 5 outlines some of the computational and conceptual problems involved in answering this question. We hope that some of our colleagues will become addicted to answering it. 


\section{References}

Auerbach, A. J., and L. J. Kotlikoff (1987). Dynamic Fiscal Policy. Cambridge University Press.

Azariadis, C., and S. Chakraborty (1998). "Agency Costs in Models of Economic Growth," forthcoming in Economic Journal.

Basu, S., and J. G. Fernald (1997). "Returns to Scale in U.S. Production: Estimates and Implications," Journal of Political Economy 105 (April): 249-83.

Benhabib, J., and R. E. A. Farmer (1994). "Indeterminacy and Increasing Returns," Journal of Economic Theory 63 (June): 19-41.

Benhabib, J., and G. Laroque (1988). "On Competitive Cycles in Productive Economies," Journal of Economic Theory 45 (June): 145-70.

Bernanke, B., and M. Gertler (1989). "Agency Costs, Net Worth, and Business Fluctuations," American Economic Review 79 (March): 14-31.

Blanchard, O.-J. (1985). "Debts, Deficits, and Finite Horizons," Journal of Political Economy 93 (April): 223-47.

Blanchard, O.-J., and D. Quah (1989). "The Dynamic Effects of Aggregate Demand and Supply Disturbances," American Economic Review 79 (September): 655-73.

Boldrin, M. (1989). "Paths of Optimal Accumulation in Two-Sector Models," in W. A. Barnett, J. Geweke, and K. Shell, eds., Economic Complexity: Chaos, Sunspots, Bubbles, and Nonlinearity, pp. 231-52. Cambridge University Press.

Boldrin, M., and L. Montrucchio (1986). "On the Indeterminacy of Capital Accumulation Paths," Journal of Economic Theory 40 (October): 26-39. 
Burnside, C.; M. Eichenbaum; and S. Rebelo (1993). "Labor Hoarding and the Business Cycle," Journal of Political Economy 101 (April): 245-73.

Calvo, G. A. (1978). "On the Indeterminacy of Interest Rates and Wages with Perfect Foresight," Journal of Economic Theory 19 (December): 321-37.

Christiano, L. J., and M. Eichenbaum (1992). "Current Real-Business-Cycle Theories and Aggregate Labor-Market Fluctuations," American Economic Review 82 (June): 43050.

Cogley, T., and J. M. Nason (1995). "Output Dynamics in Real-Business-Cycle Models," American Economic Review 85 (June): 492-511.

Gali, J. (1996). "Multiple Equilibria in a Growth Model with Monopolistic Competition," Economic Theory 8 (August): 251-66.

Grandmont, J. M. (1985). "On Endogenous Competitive Business Cycles," Econometrica 53 (September): 995-1045.

Hansen, G. D. (1993). "The Cyclical and Secular Behaviour of the Labour Input: Comparing Efficiency Units and Hours Worked," Journal of Applied Econometrics 8 (JanuaryMarch): 71-80.

Huang, H.; S. İmrohoroğlu; and T. J. Sargent (1997). "Two Computations to Fund Social Security," Macroeconomic Dynamics 1 (1): 7-44.

Kehoe, T. J.(1985). "Multiplicity of Equilibria and Comparative Statics," Quarterly Journal of Economics 100 (February): 119-47.

Kehoe, T. J.; D. Levine; A. Mas-Colell; and M. Woodford (1991). "Gross Substitutability in Large-Square Economies," Journal of Economic Theory 54 (June): 1-25.

Kydland, F. E., and E. C. Prescott (1982). "Time to Build and Aggregate Fluctuations," 
Econometrica 50 (November): 1345-70.

Laitner, J. (1990). "Tax Changes and Phase Diagrams for an Overlapping Generations Model," Journal of Political Economy 98 (February): 193-220.

Nelson, C. R., and C. I. Plosser (1982). "Trends and Random Walks in Macroeconomic Time Series: Some Evidence and Implications," Journal of Monetary Economics 10 (September): 139-62.

Ramey, G., and J. Watson (1997). "Contractual Fragility, Job Destruction, and Business Cycles," Quarterly Journal of Economics 112 (August): 873-911.

Reichlin, P. (1986). "Equilibrium Cycles in an Overlapping Generations Economy with Production," Journal of Economic Theory 40 (October): 89-102.

Ríos-Rull, J.-V. (1996). "Life-Cycle Economies and Aggregate Fluctuations," Review of Economic Studies 63 (July): 465-89.

Rotemberg, J. J., and M. Woodford (1996). "Real-Business-Cycle Models and the Forecastable Movements in Output, Hours, and Consumption," American Economic Review 86 (March): 71-89.

Samuelson, P. A. (1939). "Interactions between the Multiplier Analysis and the Principle of Acceleration," Review of Economics and Statistics 21 (May): 75-78.

Sims, C. A. (1980). "Comparison of Interwar and Postwar Business Cycles: Monetarism Reconsidered," American Economic Review 70 (May): 250-57.

Weil, P. (1989). "Overlapping Families of Infinitely-Lived Agents," Journal of Public Economics 38 (March): 183-98. 


\section{Appendix}

\section{A. Eigenvalues a of three-period endowment economy}

Uniqueness of the steady state $\bar{R}$ follows from the monotonicity of the expression $R-\gamma e_{2} / f(R, R)$, which is derived from equation (13). Similarly, we show that $\bar{R}^{2}>\gamma e_{2} / A$. This means that $\pi$ has a positive discriminant, $\pi(-1)=1+T+D<0$, and that there are two negative eigenvalues which straddle -1 , as asserted in the text.

\section{B. Proof of Lemma 1}

We show that asset demand $\alpha_{\nu}(t)$ by cohort $\nu$ at time $t=\nu, \ldots, \nu+T$ is increasing in the interest factor $R_{t}$. To do this, we ask how $\alpha_{\nu}(t)$ reacts when we replace the price vector $\left(p^{\nu}\right)=\left(p_{\nu}, \ldots, p_{\nu+T}\right)$ with $\left(\tilde{p}^{\nu}\right)=\left(\lambda p_{\nu}, \ldots, \lambda p_{t}, p_{t+1}, \ldots, p_{\nu+T}\right)$ or with $\left(\tilde{p}^{\nu}\right)=$ $\left(p_{\nu}, \ldots, p_{t}, p_{t+1} / \lambda, \ldots, p_{\nu+T} / \lambda\right)$ for some fixed $\lambda>1$. Each of these substitutions raises the price ratio $R_{t}=p_{t} / p_{t+1}$ while keeping all other price ratios constant. Weak gross substitutability means that the consumption vectors $\left(c^{\nu}, \hat{c}^{\nu}, \tilde{c}^{\nu}\right)$ corresponding to these price systems will satisfy

$$
\hat{c}_{\nu}(s) \geq c_{\nu}(s)
$$

for $s=t+1, \ldots, \nu+T$ and

$$
\tilde{c}_{\nu}(s) \leq c_{\nu}(s)
$$

for $s=1, \ldots, t$. From these inequalities and the asset accumulation identity in equation (24), we obtain

$$
\hat{c}_{\nu}(s)-c_{\nu}(s)=R_{s-1}\left[\hat{\alpha}_{\nu}(s-1)-\alpha_{\nu}(s-1)\right]-\left[\hat{\alpha}_{\nu}(s)-\alpha_{\nu}(s)\right] \geq 0
$$


for $s=t+1, \ldots, \nu+T$ and

$$
\tilde{c}_{\nu}(s)-c_{\nu}(s)=R_{s-1}\left[\tilde{\alpha}_{\nu}(s-1)-\alpha_{\nu}(s-1)\right]-\left[\tilde{\alpha}_{\nu}(s)-\alpha_{\nu}(s)\right] \leq 0
$$

for $s=\nu, \ldots, t$. Initial wealth is zero, and rational consumption requires terminal wealth to be zero as well; that is,

$$
\alpha_{\nu}(\nu+T)=\hat{\alpha}_{\nu}(\nu+T)=\alpha_{\nu}(\nu-1)=\tilde{\alpha}_{\nu}(\nu-1)=0 .
$$

Inserting (34) into (32) and (33) leads to

$$
\hat{\alpha}_{\nu}(s) \geq \alpha_{\nu}(s)
$$

for $s=t+1, \ldots, \nu+T$ and

$$
\tilde{\alpha}_{\nu}(s) \geq \alpha_{\nu}(s)
$$

for $s=\nu, \ldots, t$. Taken together, these two inequalities show that an increase in $R_{t}$ raises the entire asset profile of cohort $\nu=t-T, \ldots, t$. 
A note to word processing: The figures listed below must remain in the same folder as the paper in order for them to print in this document.

F1324400.wmf

F1324701.wmf

F1GCL500.wmf

FiGCLA01.wmf

Fig1.wmf

Fig10.wmf

Fig2.wmf

Fig3.wmf

Fig30.wmf

Fig4.wmf

Fig40.wmf

Fig5.wmf 